\title{
Traduire
}

Une autre perspective sur r tr traduction

Revue française de la traduction

$243 \mid 2020$

Les arts du spectacle

\section{Recette de traduction pour un spectacle d'humour}

\author{
Marie-Laure Faurite
}

\section{(2) OpenEdition \\ Journals}

Édition électronique

URL : http://journals.openedition.org/traduire/2227

DOI : 10.4000/traduire.2227

ISSN : 2272-9992

Éditeur

Société française des traducteurs

Édition imprimée

Date de publication : 15 décembre 2020

Pagination : 112-118

ISSN : 0395-773X

\section{Référence électronique}

Marie-Laure Faurite, «Recette de traduction pour un spectacle d'humour », Traduire [En ligne], 243 |

2020, mis en ligne le 15 décembre 2020, consulté le 31 décembre 2020. URL : http://

journals.openedition.org/traduire/2227 ; DOI : https://doi.org/10.4000/traduire.2227 


\section{Recette}

\section{de traduction pour un spectacle d'humour}

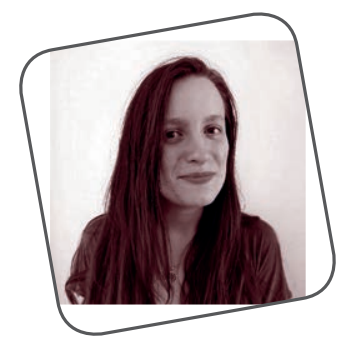

\section{Marie-Laure Faurite}

Mon expérience dans la traduction de spectacles a commencé dès le début de ma carrière, quand j'ai eu l'occasion de traduire deux spectacles des Ehrlich Brothers, deux célèbres illusionnistes et humoristes allemands. Ces artistes ambitieux avaient décidé de franchir le Rhin pour séduire le public français au Palais des Sports et au Zénith de Paris. Ma mission consistait à les aider à conquérir leur nouveau public.

Alors que la pandémie de Covid-19 a mis en pause le secteur de l'évènementiel et que tous ses travailleurs de l'ombre se retrouvent sans mission, j'ai voulu revenir sur cette expérience étonnante et tenter de mettre en avant les atouts nécessaires à la traduction d'un spectacle humoristique.

Soucieuse de la parité des genres et consciente que notre profession est en grande partie exercée par des femmes, j'ai décidé d'adopter le féminin pour mentionner notre métier ( "la traductrice» au lieu de «le traducteur»). 


\section{Plusieurs pincées de communication efficace}

\section{Comprendre le texte avant de le traduire}

Traduire un spectacle revient à partager la Weltanschauung de l'artiste, c'est-à-dire sa vision du monde. Et pour mieux comprendre la vision de l'artiste, rien de mieux qu'une communication efficace avec son client!

L'humour est intrinsèquement culturel et varie en fonction des pays, et même des régions. Ainsi, certaines blagues pourront vite sembler abstraites à quelqu'un qui n'a pas la référence culturelle adéquate, et ce quelle que soit sa maîtrise de la langue. Ce qui est drôle en allemand ne le sera pas nécessairement en français, et vice versa. Tout au long du processus de traduction et d'adaptation, il m'a semblé très important de poser des questions au client. En travaillant seulement sur un texte écrit, sans avoir les informations contextuelles (gestuelle, ton...), il est important de comprendre clairement ce qui est drôle. Nos échanges étaient, au début, ponctués de "Qu'est-ce qui est drôle dans ce passage?", "Que se passe-t-il à ce moment-là? ", "À quoi sert cette phrase? ". Certains scripts se basent d'ailleurs sur des sketchs filmés qui contiennent des passages improvisés sur le moment et qui, hors contexte, ne sont plus très clairs.

\section{S'aventurer au-delà du texte}

Évidemment, on ne peut guère traduire un spectacle en n'ayant devant soi que le texte. Un spectacle est vivant, les artistes donnent du souffle au texte et la majorité des problèmes de compréhension proviennent du manque de contexte, de gestuelle, d'expressions faciales, qui, parfois plus que le texte, suscitent le rire du public. Au début de ma mission, je n'avais que les scripts. Les sketchs me paraissaient donc très abstraits. Après que je leur en ai parlé, mes clients m'ont envoyé des supports audiovisuels, et tout est devenu plus facile à comprendre.

\section{Expliquer ses choix de traduction}

Face à son spectacle écrit dans une autre langue et dont il ne saisit pas toutes les nuances, l'artiste peut sembler 
perplexe... II est donc important de lui expliquer ce qui fait que la traduction fonctionne (pourquoi c'est drôle, pourquoi cette phrase va faire réagir le public...).

L'artiste doit rester maitre de son spectacle; il me semble donc important de ne pas lui imposer une seule traduction. En lui proposant plusieurs alternatives, je le laisse gérer son œuvre, même une fois traduite, et lui permets de mieux s'approprier ce nouveau texte.

\section{Conseiller l'artiste dans ses choix}

La traductrice est la première spectatrice de la langue cible. En tant que locutrice native, elle peut tout à fait donner son avis sur des blagues, des adaptations ou des formules qui lui semblent peu judicieuses. Les artistes m'avaient notamment demandé s'ils pouvaient plaisanter sur les «gilets jaunes», et, en 2019, je leur avais répondu que cela pouvait être hasardeux, étant donné le climat alors tendu en France. Il faut prendre en compte les différentes sensibilités et la notion de "politiquement correct», qui n'est pas forcément perçue de la même manière dans tous les pays et à laquelle n'adhèrent pas tous les artistes.

La traductrice peut également rassurer l'artiste, l'encourager sur sa prononciation, lui expliquer pourquoi il ne faut surtout pas dire certaines choses, qu'il pense innocentes, au public français. En effet, il se peut que l'artiste, s'il parle assez bien la langue cible, improvise ou brode en se souvenant des cours de langues qu'il avait à l'école. C'est ainsi que, lors des répétitions, un des magiciens a commencé à improviser et a dit vouloir "baiser une fille du public»-pour lui, «baiser» signifiait simplement "donner un baiser», et c'est sans doute ce qu'il avait appris à l'école. Il a donc fallu lui expliquer que le sens de ce verbe a évolué pour évoquer quelque chose de, somme toute, moins romantique... et ainsi éviter un éventuel scandale au Palais des Sports.

\section{Une bonne dose de créativité}

"Traduire» un spectacle d'humour s'apparente davantage à une réécriture partielle du spectacle. La mission de la traductrice est de faire en sorte que se dessinent sur les visages 
du public francophone les mêmes réactions que sur ceux du public d'origine. La meilleure alliée sera donc la créativité: nous ne faisons pas que traduire, nous réécrivons un spectacle.

\section{Se détacher du texte pour composer}

Une fois que la traductrice aura cerné les mécanismes et les stratagèmes utilisés tout au long du spectacle, ainsi que les moments où l'artiste cherche à émouvoir, impressionner ou faire rire son public, elle pourra commencer à traduire. Si les Ehrlich Brothers sont très populaires en Allemagne, ils se produisaient en France pour la première fois. II fallait donc également initier le public francophone à leur univers et à leur humour. Plus que jamais, le texte devait être percutant et efficace.

Si certains gags fonctionnent au-delà des frontières, d'autres méritent davantage de réflexion.

\section{Traduire, adapter, transcréer}

Certaines blagues doivent évidemment être adaptées, notamment celles faisant référence à des personnalités nationales, voire régionales. Dans un sketch, l'un des magiciens compare sa voix aiguë à celle de Heidi Klum, une actrice et mannequin bien connue en Allemagne. Pour évoquer une telle voix, mentionner Shirley, du célèbre duo "Shirley et Dino» me semblait plus approprié et aussi drôle pour le public français que l'allusion à Heidi Klum de la version allemande. Après que je leur ai fait écouter la voix de Shirley, les artistes ont adopté cette proposition d'adaptation.

Un bon spectacle d'humour comprend souvent des jeux de mots de qualité. Évidemment, un jeu de mots ne se traduit pas, il s'adapte. Et pour cela, il faut parvenir à se détacher du texte source pour réussir à trouver un équivalent dans la langue cible.

Dans une scène, l'un des deux artistes montre sa nouvelle veste pleine de rivets et son frère se moque de ses goûts vestimentaires douteux. Le jeu de mots qui s'ensuit se base en allemand sur la polysémie du mot Niete qui désigne à la fois les rivets des vêtements, mais également un «minable» ou un «nullos». 


\begin{tabular}{|c|c|c|}
\hline $\begin{array}{l}\text { Texte source } \\
\text { A: Alter, guck mal hier, richtig cool } \\
\text { mit Nieten. } \\
\text { B: Ja und die größte Niete ist in } \\
\text { der Jacke. }\end{array}$ & $\begin{array}{l}\text { Traduction littérale } \\
\text { A : Mais regarde, elle est vraiment } \\
\text { cool avec tous ces rivets } \\
\text { B : Oui, et le plus gros des } \\
\text { rivets/minables se trouve dans } \\
\text { la veste }\end{array}$ & $\begin{array}{c}\text { Adaptation } \\
\text { A : Mais regarde, c'est super } \\
\text { cool tous ces clous ! } \\
\text { B : Arrête, tu t'enfonces ! }\end{array}$ \\
\hline
\end{tabular}

\section{Laisser reposer la réflexion}

Dans leur spectacle, les Ehrlich Brothers utilisent souvent l'interjection allemande Hammer!; le mot seul désigne un marteau, mais, assorti d'un point d'exclamation, il signifie dans le langage courant «Génial!» ou «Super!». Les artistes le répètent tant qu'il fait désormais partie de leur identité. Ils n'hésitent pas àjouer dessus et à brandir des (faux) marteaux sur scène. Mais comment traduire cette véritable marque de fabrique?

Après leur avoir soumis différentes propositions et en avoir discuté avec eux, j'ai compris qu'ils ne recherchaient pas nécessairement quelque chose qui soit très "à la mode», mais plutôt qui soit suffisamment imagé. C'est ainsi que nous avons adopté «Chouette!» pour l'équivalent français de Hammer!, dans l'idée de faire de la chovette leur emblème en France.

Pour certains passages, une traduction satisfaisante ne vient qu'au bout de quelques jours, voire quelques semaines de recherche.

\section{Sortir la traduction du four}

Dans un tel contexte de traduction créative, la TAO n'est évidemment pas un support idéal. Il est important de reconstruire les phrases, de relire le texte à tête reposée, sur ordinateur et sur papier. Après avoir sorti la traduction du four, je la laisse toujours refroidir quelques jours... avant de la relire à haute voix.

Il est important de garder en tête que les traductions de spectacles vont être dites. Lire la version française à haute voix permet de s'assurer qu'elle fonctionne bien. On vérifie ainsi la fluidité du discours, le rythme des répliques, les enchaînements et les transitions entre les différents numéros. S'affranchir de la segmentation d'un logiciel de TAO est donc fondamental pour adapter un spectacle. 


\section{Rajouter $\mathbf{3 0 0}$ grammes de professionnalisme et de perfectionnisme}

Traduire pour la scène, c'est traduire un spectacle qui va prendre vie, au-delà des mots.

\section{S'adapter aux besoins de l'artiste}

Si l'artiste n'est pas un locuteur natif, il peut avoir besoin de l'aide de la traductrice pour savoir comment prononcer certains mots ou pour raccourcir certains passages qu'il a du mal à mémoriser. Mon rôle consiste alors à l'accompagner. J'ai transcrit certains passages délicats dans une "phonétique» qui parlait à l'artiste. II ne s'agit pas d'utiliser un alphabet phonétique officiel, mais une transcription en lien avec les codes de prononciation de sa propre langue. L'objectif était de lui faciliter la lecture des textes qui défilaient sur les prompteurs.

Voici un exemple de «transcription phonétique» à destination de l'artiste de langue natale allemande:

\section{c'est l'heure dö deeßinee pour: \\ c'est l'heure de dessiner}

\section{Pour finir: un soupçon d'audace et un esprit artistique}

La traduction est rarement finie au moment de la livraison du texte. Les modifications de dernière minute sont fréquentes.

Si l'artiste le propose, la traductrice l'accompagnera dans sa tournée internationale pour l'aider. Cela implique qu'elle soit disponible, bloque certaines dates à l'avance et prévoie parfois des prolongations de séjour au dernier moment. Elle vivra alors au rythme de l'artiste, c'est l'aventure.

Dans ce secteur, les clients apprécient que la traductrice puisse s'adapter et se montrer à son tour entreprenante et créative. Dans le dernier spectacle que j'ai traduit, l'un des frères interprète une chanson émouvante qui ne faisait pas partie du script. Je leur ai donc proposé de la traduire afin qu'ils puissent la chanter en français dans leur spectacle. 
Cet exercice permet encore une fois d'aller au-delà du texte et de faire face à d'autres défis, comme l'adéquation entre le solfège et le rythme du français.

Dans le domaine des spectacles et du divertissement, la traductrice vit les mêmes imprévus que les artistes. Et cette année, les annulations des dates et des tournées dues à la pandémie sont autant de missions qui tombent à l'eau.

\section{Conclusion}

J'ai présenté dans cet article des situations auxquelles j'ai été confrontée au cours de ma collaboration avec ces clients originaux. Un spectacle bien traduit est selon moi un spectacle qui n'a pas l'air d'avoir été traduit. Le but est de faire oublier que les artistes sont étrangers et de rire ou de s'émouvoir avec eux. La bonne traduction se situe là où se rencontrent la créativité de l'artiste et celle de la traductrice.

Je n'oublierai jamais l'effet ressenti en entendant le public rire des blagues que j'avais écrites, l'émotion d'être invitée sur scène et applaudie. Je suis vraiment reconnaissante aux Ehrlich Brothers de m'avoir permis de vivre tout cela, et j'espère avoir l'occasion de renouveler ce genre d'aventures une fois la crise sanitaire passée.

Merci à Maëva, sans qui cette aventure n'aurait pas été possible. Merci à Hilde, Chris et Andreas pour leur confiance et leur professionnalisme. Merci à Adrien, mon relecteur pour cette mission, et merci à Séverine et à Nathan pour leur soutien.

\section{contact@tradixit.com}

Marie-Laure Faurite est traductrice (anglais et allemand vers le français). Passionnée par l'art et l'écriture, titulaire d'une licence d'anglais (LLCE) et d'allemand (LLCER), elle valide en 2018 un master en traduction spécialisée multilingue à l'université Grenoble Alpes. En 2019, elle accompagne les magiciens Ehrlich Brothers dans leur tournée internationale et adaptera deux de leurs spectacles. Elle se lance ainsi dans la traduction créative et artistique pour les domaines du divertissement (spectacles, jeux de société, loisirs créatifs) sous l'entité Tradixit avec une collègue. 